\title{
Resonant transport and electrostatic effects in single-molecule electrical junctions
}

\author{
Carly Brooke, ${ }^{1}$ Andrea Vezzoli, ${ }^{1}$ Simon J. Higgins, ${ }^{1}$ Linda A. Zotti, ${ }^{2,3,}{ }^{*}$ J. J. Palacios, ${ }^{2,4}$ and Richard J. Nichols ${ }^{1}$ \\ ${ }^{1}$ Department of Chemistry, University of Liverpool, Crown Street, Liverpool L69 7ZD, United Kingdom \\ ${ }^{2}$ Departamento de Física de la Materia Condensada and Condensed Matter Physics Center (IFIMAC), \\ Universidad Autónoma de Madrid, 28049 Cantoblanco, Madrid, Spain \\ ${ }^{3}$ Departamento de Física Aplicada, Universidad de Alicante, 03690 Alicante, Spain \\ ${ }^{4}$ Instituto Nicolas Cabrera, Universidad Autónoma de Madrid, 28049 Cantoblanco, Madrid, Spain \\ (Received 27 November 2014; revised manuscript received 13 April 2015; published 26 May 2015)
}

\begin{abstract}
In this contribution we demonstrate structural control over a transport resonance in $\mathrm{HS}\left(\mathrm{CH}_{2}\right)_{n}[1,4-$ $\left.\mathrm{C}_{6} \mathrm{H}_{4}\right]\left(\mathrm{CH}_{2}\right)_{n} \mathrm{SH}(n=1,3,4,6)$ metal-molecule-metal junctions, fabricated and tested using the scanning tunneling microscopy-based $I(z)$ method. The Breit-Wigner resonance originates from one of the arene $\pi$-bonding orbitals, which sharpens and moves closer to the contact Fermi energy as $n$ increases. Varying the number of methylene groups thus leads to a very shallow decay of the conductance with the length of the molecule. We demonstrate that the electrical behavior observed here can be straightforwardly rationalized by analyzing the effects caused by the electrostatic balance created at the metal-molecule interface. Such resonances offer future prospects in molecular electronics in terms of controlling charge transport over longer distances, and also in single-molecule conductance switching if the resonances can be externally gated.
\end{abstract}

DOI: 10.1103/PhysRevB.91.195438

PACS number(s): 73.63.Rt, 73.40.Sx, 85.65.+h

\section{INTRODUCTION}

The development of reliable techniques for the fabrication and electrical testing of metal-single-molecule-metal junctions, supported by rapid developments in theoretical treatment of such junctions, has led to a significant re-invigoration of the wider field of molecular electronics [1-5]. The techniques used can be divided into two categories. One category uses solid-state electronics technology to fabricate so-called break junctions, in which a very thin metallic wire is deliberately broken (either by electromigration [6] or by the application of mechanical stress [7]) in such a way that a molecule can bridge the resulting gap. The other category involves the use of scanning probe microscopy to fabricate and characterize metal-molecule-metal junctions. For example, in the scanning tunneling microscopy break junction (STM-BJ) method, a gold STM tip is driven into a gold substrate, and then retracted while the current is monitored [8]. As the resulting chains of gold atoms are broken, step decreases in the current approximately corresponding to the quantum of conductance $G_{0}$ are seen until the final chain of gold atoms is broken. In the presence of molecules that contain terminal potential binding groups (e.g., thiols [8], pyridines [8-10], amines [11,12], carboxylate anions [11,13], phosphines [14,15], thioethers [14]), additional much smaller current step decreases are seen after the final gold atomic chain breaks, and these correspond to the subsequent breaking of metal-molecule-metal junctions. Alternatively, in the so-called $I(z)$ ( $I$, current; $z$, vertical height) method for measuring single-molecule electrical properties, a gold STM tip is brought into close proximity of a surface coated with suitable molecules without allowing it to come into contact, and is then retracted while the tunneling current is monitored [16]. When a molecule bridges the gap prior to tip retraction, a characteristic plateau is seen in the currentdistance curve, prior to junction breakdown whereupon the current drops sharply.

*Corresponding author: linda.zotti@uam.es
For the great majority of (relatively short) molecules that have been studied in metal-single-molecule-metal junctions, the mechanism of conductance has been found to be coherent tunneling [1]. The conductance $G_{M}$ then varies as a function of the length of the bridging molecule according to Eq. (1):

$$
G_{M}=G_{\mathrm{con}} e^{-\beta R},
$$

where $G_{\text {con }}=G_{0} T_{L} T_{R}$ ( $G_{0}$ is the quantum unit of conductance, $77.4 \mu \mathrm{S}$, and $T_{L}$ and $T_{R}$ are the transmittances of the left-hand and right-hand connections of the molecule to the metal contacts; $G_{\text {con }}$ is called the contact conductance and represents the conductance of the system in the absence of the molecular backbone), $R$ is the molecular backbone length, and $\beta$ is the attenuation factor. A plot of $\ln G_{M}$ vs $R$ is used to determine $\beta$. Often, such molecules can be considered as "passive" molecular wires, in which the conductance decreases with bridge length in the simple manner predicted by phase coherent tunneling. In many cases this length decay can be straightforwardly linked to the degree of conjugation, in turn related to the proximity of the relevant highest occupied molecular orbital (HOMO) or lowest unoccupied molecular orbital (LUMO) ("frontier") orbitals to the metal contact Fermi energies $E_{F}$. However, molecular electronics is now aiming to achieve more active control over the electrical properties of molecular bridges. Approaches to this include electrochemical switching or conformational control of bridge structure and electronic states and control of quantum interference in molecular circuits.

In earlier work, we showed that the conductance of junctions involving $6 \mathrm{Ph} 6$ and its derivatives (Fig. 1) was much higher $(0.7 \mathrm{nS})$ than that measured for 1,12-dodecanedithiol (i.e., the two alkyl links for $6 \mathrm{Ph} 6$ connected back to back; $0.028 \mathrm{nS}$ [17]), even though $6 \mathrm{Ph} 6(2.10 \mathrm{~nm} \mathrm{~S} \ldots \mathrm{S}$ distance $)$ is considerably longer than 1,12-dodecanedithiol $(1.72 \mathrm{~nm}$ S ...S). We suggested that the reason for the unusually high conductance of $6 \mathrm{Ph} 6$ was that it behaves as a "molecular double tunneling barrier" in which a "well" with lower HOMO-LUMO $\left(\pi-\pi^{*}\right)$ separation is sandwiched between two 
(a)
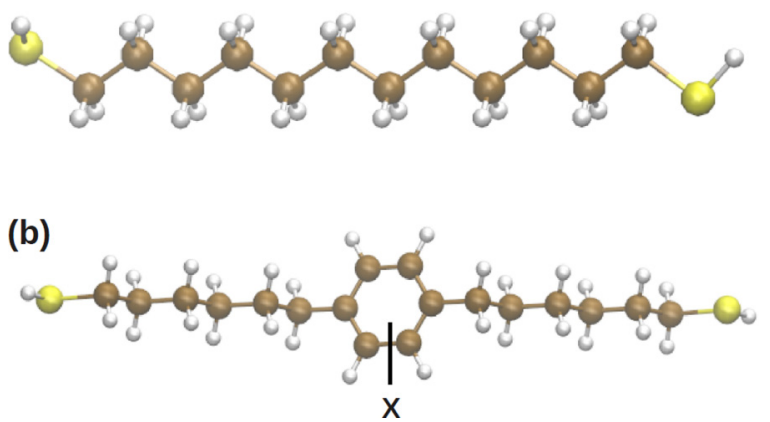

FIG. 1. (Color online) Structural comparison of (a) 1,12dodecanedithiol with (b) $6 \mathrm{Ph} 6$ and its derivatives $(X=2,3,5,6$ tetrafluoro-, 2,5-dimethyl, 2,5-dimethoxy-).

"barriers" with higher HOMO-LUMO $\left(\sigma-\sigma^{*}\right)$ separation, by analogy with inorganic double tunneling barrier structures involving III-V semiconductors [18]. Clearly, the benzene moiety is capable of acting as an indentation in the tunneling barrier. Interestingly, control over the conductance of the $6 \mathrm{Ph} 6$ family could be exerted chemically by using substituents on the aryl ring (Fig. 1); electron-donating substituents ( $\mathrm{Me}-$, $\mathrm{MeO}-$ ) resulted in significantly higher junction conductances and the electron-deficient $\mathrm{C}_{6} \mathrm{~F}_{4}$ group resulted in a lower conductance [18]. Many studies of metal-molecule-metal junctions have probed the molecular structural requirements for obtaining high junction conductance [19-28].

The work described in this contribution is aimed at a deeper understanding of the evolution of the orbital alignment in single-molecule junctions. To this end, we have investigated the effect of varying the width of the barriers (i.e., the number of methylene groups) while keeping the well (the 1,4- $\mathrm{C}_{6} \mathrm{H}_{4}$ moiety) constant. Accordingly, we have now synthesised additional molecules and have determined the single-molecule conductances of 1,4- $\mathrm{HS}\left(\mathrm{CH}_{2}\right)_{x} \mathrm{C}_{6} \mathrm{H}_{4}\left(\mathrm{CH}_{2}\right)_{x} \mathrm{SH}(x \mathrm{Phx} ; x=1$, $3,4,6)$ using the $I(z)$ technique, and we have carried out transport calculations on the molecules. We find that the variation of molecular conductance with $n$ in this family is surprisingly small, and that this is the consequence of the presence of a Breit-Wigner resonance in the transmission curve (caused by the higher energy of the two $\pi$-bonding orbitals of the benzene ring). This resonance sharpens and moves closer to the contact Fermi energy as $n$ increases, offsetting to some degree the rapid exponential decrease in conductance with length that might be expected for increasing $n$. Thus, control over the conductance of these molecules can be exerted not only by substituents on the phenyl ring, but also by varying the alkyl chain length. More importantly, we show that this system can be understood in terms of a simple pair of back-to-back metal-semiconductor junctions where the alignment of the states in the internal part of the semiconductor primarily depends on how the electrostatic balance is established at the contact region. Such a balance is in turn mainly determined between the molecular midgap states and the metal Fermi level via charge transfer, which finally determines the position of the bulk resonance with respect to the Fermi level. We also show that investigating this system within this perspective is essential to understand the level alignment in the junction, (a)
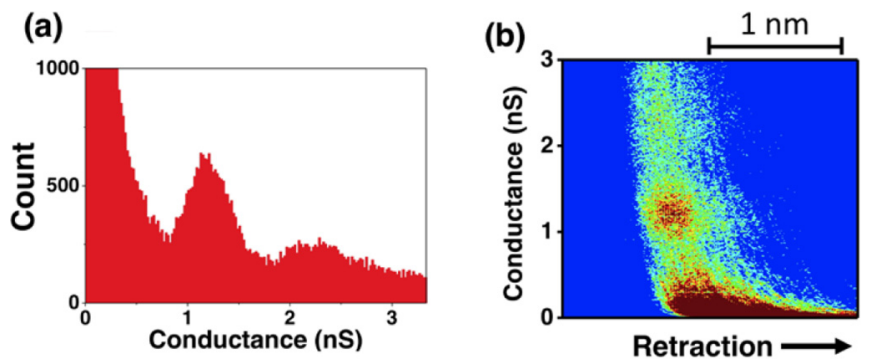

FIG. 2. (Color online) (a) Histogram of $500 I(s)$ scans obtained for $4 \mathrm{Ph} 4$ using a set point current of $10 \mathrm{nA}$, and (b) a two-dimensional histogram plot, color-coded to show frequency of occurrence of conductance as a function of distance in these $I(s)$ traces.

which otherwise could not be fully explained by the initial trend in the gas phase.

\section{EXPERIMENTAL RESULTS}

\section{A. Syntheses, sample preparation, and monolayer formation}

$1 \mathrm{Ph} 1$ (as the free dithiol) is commercially available and was used as received. $6 \mathrm{Ph} 6$ (dithioacetate; Fig. 1) was synthesised as previously described [18] and the same general approach was used to synthesise $3 \mathrm{Ph} 3$ and $4 \mathrm{Ph} 4$; full details are provided in the Supplemental Material (SM) [29]. Gold-coated glass substrates (Arrandee) of approximately $1 \times 1 \mathrm{~cm}$ size were flame-annealed using a butane-air torch to red heat, to generate $\mathrm{Au}(111)$ terraces [30], as confirmed by STM imaging (see the SM [29], Fig. 1). The slides were allowed to cool, and were then immersed in a $10^{-3} \mathrm{~mol} \mathrm{dm}^{-3}$ solution of the appropriate dithioacetate ( $3 \mathrm{Ph} 3,4 \mathrm{Ph} 4,6 \mathrm{Ph} 6)$ or dithiol (1Ph1) in $\mathrm{CH}_{2} \mathrm{Cl}_{2}$ for $1-2 \mathrm{~min}(1 \mathrm{Ph} 1 ; 3 \mathrm{~min})$ to afford a partial monolayer coverage of adsorbed dithiol molecules. Polarization modulated infrared reflection-absorption spectroscopy (PM-IRRAS) was used to confirm that the adsorption of the molecules took place (for an example, see Fig. 2 in the SM), and STM imaging of these submonolayers showed bright spots indicative of the presence of molecule(s), particularly at step edges [see the SM [29], Fig. 1(c)]; molecular resolution could not be obtained, as is typical for highly mobile adsorbed thiols at submonolayer coverages.

\section{B. Single-molecule conductance determination}

The scanning tunneling microscopy-based $I(z)$ technique was used to determine single-molecule conductance values for $1 \mathrm{Ph} 1,3 \mathrm{Ph} 3,4 \mathrm{Ph} 4$, and $6 \mathrm{Ph} 6$. In this technique, tip-substrate contact is avoided. A freshly cut Au STM tip was brought into close proximity to the modified substrate surface using the appropriate set point current, and the tip was then retracted while the tunneling current was measured. When a molecule (or molecules) bridge between tip and substrate, characteristic current plateaux in the current-vertical distance $(I-z)$ traces result, and the molecular conductance is determined from a histogram of many such traces. In this work, at least 500 $I-z$ traces showing current plateaux (defined as having length $\geqslant 0.5 \mathrm{~nm}$ ) were combined into conductance histograms for any given experiment; typically, approximately $15 \%$ of $I-z$ traces showed a plateau. Of the remaining traces, approximately $60 \%$ 
(a)

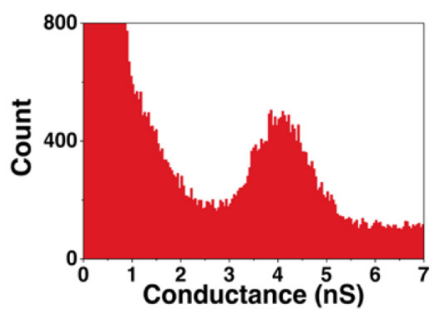

(b)

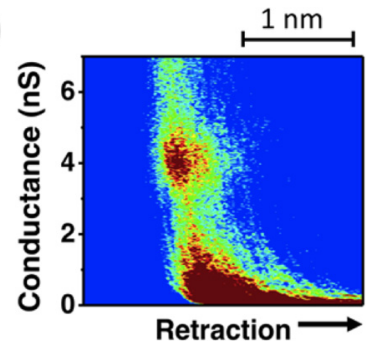

FIG. 3. (Color online) (a) Histogram of $500 I(s)$ scans obtained for $1 \mathrm{Ph} 1 \mathrm{using}$ a set point current of $30 \mathrm{nA}$, and (b) a two-dimensional histogram plot, color-coded to show frequency of occurrence of conductance as a function of distance in these $I(s)$ traces.

were straightforward exponential decays with no evidence of molecular bridge formation and the rest were noisy and showed no obvious plateau; $I-z$ traces in these categories were rejected. Two-dimensional histograms of conductance-vertical distance correlation were also constructed. The tip bias in all experiments was $+0.6 \mathrm{~V}$.

Initially, we began by repeating the experiment of Leary et al. [18] using 6Ph6, with a set point current of $6 \mathrm{nA}$. This produced a relatively sharp histogram peak at a conductance of $0.82 \pm 0.18 \mathrm{nS}$, in good agreement with the results reported by Leary et al. (see the SM [29]; Fig. 4). A second clear peak can also be seen at approximately twice this value, corresponding to junctions formed with two molecules in the gap. Next, we examined $4 \mathrm{Ph} 4,3 \mathrm{Ph} 3$, and $1 \mathrm{Ph} 1$. It was necessary to increase the set point current for these molecules, to 10,20 , and $30 \mathrm{nA}$ respectively, since the molecules are progressively shorter, hence the tip has to be brought into closer proximity to the surface to ensure a reasonable percentage of successful junction formation events on tip retraction. Figure 2 shows the results obtained for $4 \mathrm{Ph} 4$, and Fig. 3 those for $1 \mathrm{Ph} 1$; corresponding results for $3 \mathrm{Ph} 3$ are shown in Fig. 3 in the SM [29]. A small proportion of the $I-z$ traces from these experiments showed some evidence of a final, lower-conductance plateau, following the plateau that gave rise to the above conductance peaks. This suggested that lower-conductance junctions might form with these molecules at larger tip-substrate distances, but these plateaux do not give rise to a discernible separate conductance peak in either one-dimensional or two-dimensional histograms [see, e.g., Figs. 2(a) and 2(b) where they are lost in the low-current noise at $<0.8 \mathrm{nS}]$. These events were quite rare, even when the set-point current was set to a smaller value to favor the formation of these longer, lower conductance plateaus over the shorter, higher conductance events, and so we did not analyze these events further. The data for all experiments are compared in Table I. We used a previously published procedure to measure the break-off distance (the distance $z$ at which the junctions break down) [26] and to correct this for the initial set-point distance $z_{0}$ (the initial height of the STM tip). These break-off distances were similarly plotted as histograms (see the SM [29], Fig. 6). Since, as is evident from Figs. 2 and 3, the conductances of the junctions are rather invariant with distance for these highly flexible molecules, we use the 95th percentile break-off distance as an estimate of the maximum

TABLE I. Summary of conductance data for molecules xPhx.

\begin{tabular}{lccc}
\hline \hline & \multicolumn{2}{c}{$\begin{array}{c}\text { Conductance } \\
\text { Molecule }\end{array} \quad \begin{array}{c}\text { 95th percentile } \\
\text { break-off distance }(\mathrm{nm})\end{array}$} & $\begin{array}{c}\text { Calcd. Au } \ldots \mathrm{Au} \\
\text { distance for } \mathrm{Au}-x \mathrm{Ph} x \\
\text {-Au junction }(\mathrm{nm})^{\mathrm{a}}\end{array}$ \\
\hline $1 \mathrm{Ph} 1$ & $4.14 \pm 0.58$ & 1.4 & 1.1 \\
$3 \mathrm{Ph} 3$ & $2.01 \pm 0.32$ & 1.8 & 1.7 \\
$4 \mathrm{Ph} 4$ & $1.21 \pm 0.19$ & 1.9 & 1.9 \\
$6 \mathrm{Ph} 6$ & $0.82 \pm 0.18$ & 2.5 & 2.3 \\
\hline \hline
\end{tabular}

${ }^{\mathrm{a}}$ Calculated for the fully extended (all- anticonformer) molecule, terminated by two Au-S bonds, using Spartan 14 molecular mechanics calculations.

length of the junction at breakdown, and it can be seen from Table I that this correlates well with the theoretical length of a junction between the two contacting gold atoms, approximated for the fully extended molecular conformation using molecular mechanics (Spartan 14). The exception is $1 \mathrm{Ph} 1$, where the junctions at breakdown are somewhat longer than expected for a molecule of this length. It is possible that for a very short molecule, extension of the junction results in gold atoms being pulled out from the surface prior to junction breakdown.

It is interesting to note that among the molecules studied here, only $1 \mathrm{Ph} 1$ has previously been studied by the STM-BJ technique, and at $0.6 \mathrm{~V}$ bias voltage, the conductance measured was $80 \mathrm{nS}$ [31]. It has previously been shown that the STM-BJ method favors the formation of high conductance molecular junctions, and this has been interpreted in terms of the contact atoms binding to gold at step edges or similar defects, producing a junction with a shorter metal-metal distance and with additional channels for conduction through the molecule due to the step edge gold atom in close proximity to the molecular backbone [17]. In Fig. 4, we compare the

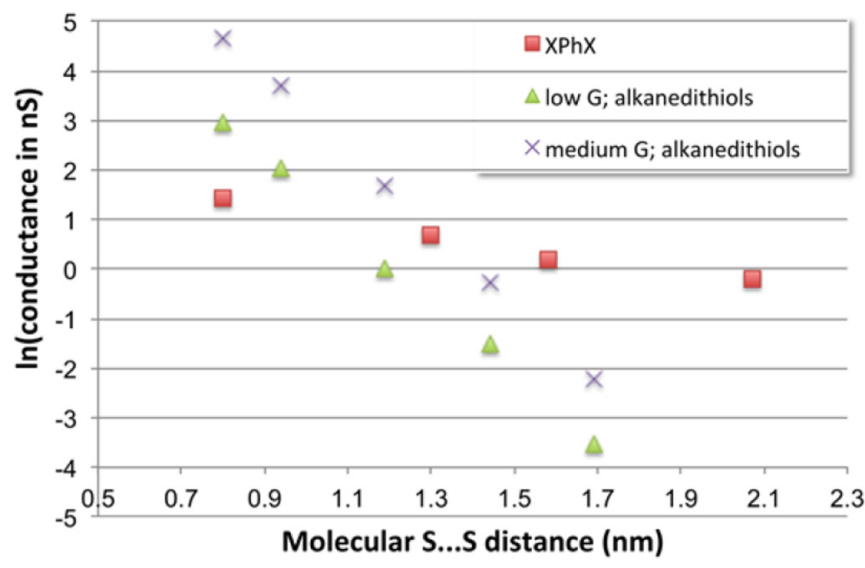

FIG. 4. (Color online) Plots of $\ln$ (conductance in $\mathrm{nS}$ ) vs molecular length between the contact $\mathrm{S}$ atoms for the $x \mathrm{Ph} x$ series (this work), and for the low and medium conductance groups as recently measured for 1,5-pentanedithiol, 1,6-hexanedithiol, 1,8octanedithiol, and 1,10-decanedithiol in UHV [32] and in ambient (1,12-dodecanedithiol [21]). These examples are shown as these molecules have similar lengths to the shorter members of the $x \mathrm{Ph} x$ series; the conductances of junctions with longer alkanedithiols have not been experimentally determined in this fashion as the conductance values would be too low. 
conductance as a function of length between the sulfur contact atoms (calculated for the fully extended molecules using the molecular mechanics approach in Spartan14) for the $x \mathrm{Ph} x$ series with recent data for the alkanedithiols, measured in UHV [32]. A notable feature of the conductance data for the $x \mathrm{Ph} x$ series is the very low apparent value of $\beta$ [calculated from the slopes of these lines; Eq. (1)], $1.52 \mathrm{~nm}^{-1}$.

Since the only structural change in the molecules $x \mathrm{Ph} x$ is the length of the alkyl chains, it is pertinent to compare the $\beta$ values with those found for alkanedithiols in related STM-based experiments; reported values range $7-8.2 \mathrm{~nm}^{-1}$ for all three conductance groups $[17,33,34]$. Since the Fermi energy of the contacts has to fall between the HOMO and LUMO of the molecule in the junction, for molecules conducting via a tunneling mechanism one expects the $\beta$ value to depend upon the HOMO-LUMO separation, with more conjugated molecules having a smaller HOMO-LUMO gap and hence a smaller $\beta$. For instance, for a series of oligophenyl methanethiols $\left(\mathrm{C}_{6} \mathrm{H}_{5} \mathrm{CH}_{2} \mathrm{SH}\right.$, $\mathrm{C}_{6} \mathrm{H}_{5} \mathrm{C}_{6} \mathrm{H}_{4} \mathrm{CH}_{2} \mathrm{SH}$, and $\mathrm{C}_{6} \mathrm{H}_{5} \mathrm{C}_{6} \mathrm{H}_{4} \mathrm{C}_{6} \mathrm{H}_{4} \mathrm{CH}_{2} \mathrm{SH}$ ), $\beta$ was determined as $4.6 \pm 0.7 \mathrm{~nm}^{-1}$ using the $\mathrm{GaIn} / \mathrm{Ga}_{2} \mathrm{O}_{3}$ eutectic contact technique on self-assembled monolayers of the molecules on template-stripped gold electrodes [35] (a similar value, $4.2 \pm 0.7 \mathrm{~nm}^{-1}$, was determined earlier using a conducting AFM technique [36]), while for a series of oligothiophenes with 5, 8, 11, and 14 2,5-coupled thiophene rings, $\beta$ was $1 \mathrm{~nm}^{-1}$, measured by the STM break junction method [21]. The lowest $\beta$ values so far measured have been for extended viologens $\left(0.06 \mathrm{~nm}^{-1}\right)$ [37] and for conjugated fused oligoporphyrins, oligoporphyrin-ethynylene, and oligoporphyrinbutadiynylene systems, where values of $0.2-0.4 \mathrm{~nm}^{-1}$ have been found, the exact value depending on structure [24-27]. It should be noted that although a low value of $\beta$ is often taken as an indication that the conductance mechanism could be hopping rather than superexchange (tunneling), it has been shown that the conductance behavior of even the most conjugated of these families of porphyrins as a function of temperature are still described well by theoretical models involving tunneling [24]. In this context, a value of $\beta$ of approximately $1.5 \mathrm{~nm}^{-1}$ is remarkably small for the $x \mathrm{Ph} x$ series, given that the only variable in this series is the number of methylene groups, for which we would expect $\beta$ to be $7-8 \mathrm{~nm}^{-1}$. To check for a possible hopping mechanism, we have carried out conductance measurements as a function of temperature on a representative molecule from this series, 6Ph6 (for data, see Fig. 5 in the SM [29]). The conductance of this molecule as determined by the $I(z)$ technique did not vary, within experimental limits, over the temperature range $20-100^{\circ} \mathrm{C}$. This confirms that a hopping mechanism is not operative, as expected since a single benzene ring is not redox-active within conventional potential limits.

\section{THEORETICAL MODELING AND DISCUSSION}

To gain insight into the conductance behavior of the $x \mathrm{Ph} x$ system we performed density-functional theory (DFT) and electronic transport calculations with the help of the code Gaussian [38], using the PBE functional [39] and the LANL2DZ basis set [40], unless otherwise specified. The molecular junctions were built by matching the $\mathrm{S}$ atoms of the

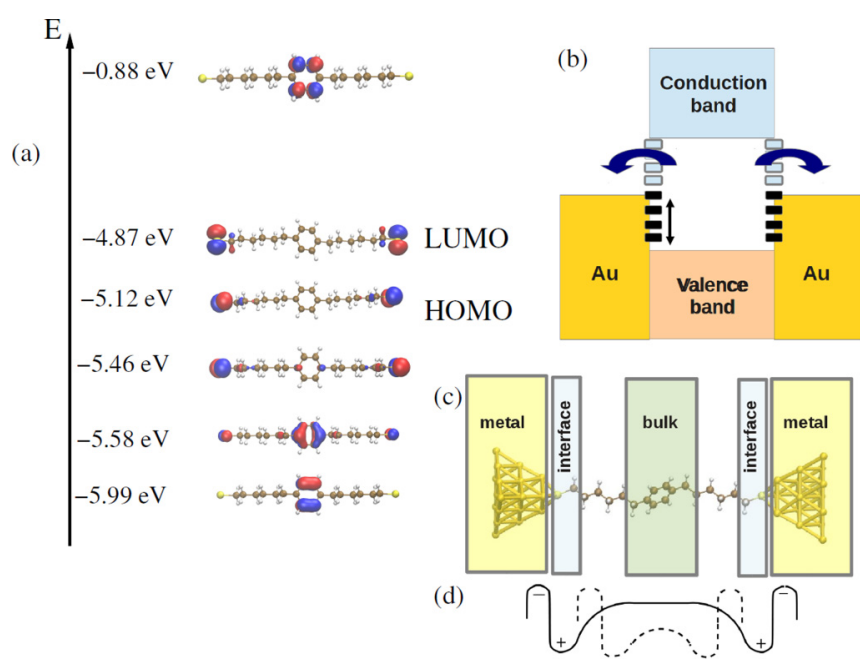

FIG. 5. (Color online) (a) Frontier orbitals of 6Ph6; the $\mathrm{H}$ atoms of the thiols are omitted as these are lost on adsorption to gold. (b) Schematic picture of the metal-semiconductor-metal junction created once the molecule is connected to the electrodes. The vertical arrow indicates the energy difference between the metal Fermi level and the valence band. (c) A model of the $6 \mathrm{Ph} 6$ molecule in a junction, forming a back-to-back pair of metal-semiconductor interfaces where the benzene ring is treated as the semiconductor bulk, the gold contacts are the metal, and the $\mathrm{S}$ and outermost $\mathrm{C}$ atoms represent an interface region. (d) Schematic electronic potential along the molecular junction depicted in panel (c) (solid line) and along a junction comprising a shorter molecule (dashed line). In the case of the longer molecule, the potential generated by the two point dipoles at the interfaces gradually vanishes in the bulk region, which is therefore only partially affected by the presence of the dipoles. This is not the case for the shorter molecule, which does not offer enough distance for this recovery and where the effect of the dipoles in the bulk region is stronger.

molecule with the $\mathrm{S}$ atoms of the S-Au structure. The electronic transport calculations were carried out with ANT.G [41,42], which is built as an interface to Gaussian. This code computes the electronic transmission using nonequilibrium Green'sfunction techniques in the spirit of the Landauer formalism, employing parametrized tight-binding Bethe lattices in the far electrode description [42]. A CRENBS basis set was used [43] for the Au atoms in the outermost layers. Further details of the calculations involving alternative contact sites (top, step) are given in the SM [29]. We optimized the molecular structures for the geometries of $1 \mathrm{Ph} 1,3 \mathrm{Ph} 3,4 \mathrm{Ph} 4$, and $6 \mathrm{Ph} 6$ in the gas phase and then relaxed a $\mathrm{S}$ atom on top of a 19-gold-atom cluster (together with the gold atoms in the top layer). Then we analyzed their electronic structure after removing the terminal $\mathrm{H}$ atoms, which are known to be lost upon adsorption onto gold under our conditions [44].

For all molecules, the HOMO-2 is localized on the benzene ring and is found to rise in energy as the chain length increases (see Fig. 9). As will be seen later in the discussion, the length dependence of the alignment of this orbital with the Fermi energy plays a determining role in the conductance behavior. The LUMO, HOMO, and HOMO-1 all consist of spin degenerate levels, localized on the $\mathrm{S}$ atoms. Orbitals localized on the alkyl chains are far below in energy. In the left 
panel of Fig. 5, we show the orbitals for the longest molecule (6Ph6) with the corresponding energies. It can be observed that the LUMO (localized on the $\mathrm{S}$ atoms) is quite close in energy to the HOMO, while the LUMO+1 (localized on the benzene) is far above. More importantly, a clear spatial separation appears between the states localized on the $\mathrm{S}$ atoms and those localized on the benzene ring.

In our view, the LUMO+1 and HOMO- 2 can be likened to the conduction-valence-band structure in a semiconductor, while the states in between (LUMO, HOMO, and HOMO-1) can be likened to the surface midgap states of the semiconductor as they are mainly localized on the $\mathrm{S}$ atoms that connect to the electrodes. Consequently, placing this molecule between two metal electrodes will form a junction that is expected to behave like a pair of back-to-back metal-semiconductor junctions in which the chemical terminations of the semiconductor create states within its band gap [as schematically depicted in Fig. 5(b)]. For the purposes of this analogy we refer to the benzene core of the molecule as the "semiconductor bulk." The whole junction can then be thought of as divided into five main blocks: metal-interface-bulk-interface-metal [Fig. 5(c)]. Each diode consists of a metal-(organic) semiconductor pointlike junction. In our case, the semiconductor is intrinsic and the metal Fermi level falls between the HOMO and the LUMO of the central benzene ring (i.e., between the HOMO-2 and the LUMO+ 1 of the complete system).

It is not straightforward, a priori, to know where the Fermi level will lie in this gap. However, insights can be gained by considering what happens at the interface between a metal and a semiconductor. In analogy with the semiconductor surface states, the states localized on the $\mathrm{S}$ atoms of the $x \mathrm{Ph} x$ molecules should be strongly hybridized when interacting with the metal, giving rise to the so-called "gateway states" [45]. The mismatch between the metal Fermi level and the molecular charge neutrality level (CNL) at the interface induces a charge transfer which in turn creates a pointlike dipole [46]; the energy levels in the inner part of the semiconductor bulk are then shifted up or down due to the dipole by a quantity which varies with the distance from the interface [Fig. 5(d)]. The role of dipole interfaces has been extensively investigated in the case of organic monolayers or (more generally) single molecules lying flat on surfaces [47-50] but, to the best of our knowledge, a thorough study concerning their roles in junctions comprising a single molecular nanowire held between two metal clusters has not been explored to the same extent [51-53]. It is worth stressing that in this case, since the semiconductor is intrinsic, no depletion layer is formed. Consequently, variations in the energy position of the bulk levels must not be ascribed to the typical band bending associated with it in doped semiconductors. Here, the only electrostatic effect is related with the dipole established at the interface and will be confined to a narrow region close to it. Notice also that while in a planar junction the bands are shifted at (infinitely) large distances [54], in a single-point junction, such as the one considered here, the effect of the dipole effect is expected to decay rapidly along the transport direction. In what follows, we proceed to analyze the energetics of the four molecules when placed between two gold electrodes; we will show how studying the electrostatic balance established at the metal-molecule interface is essential for a thorough

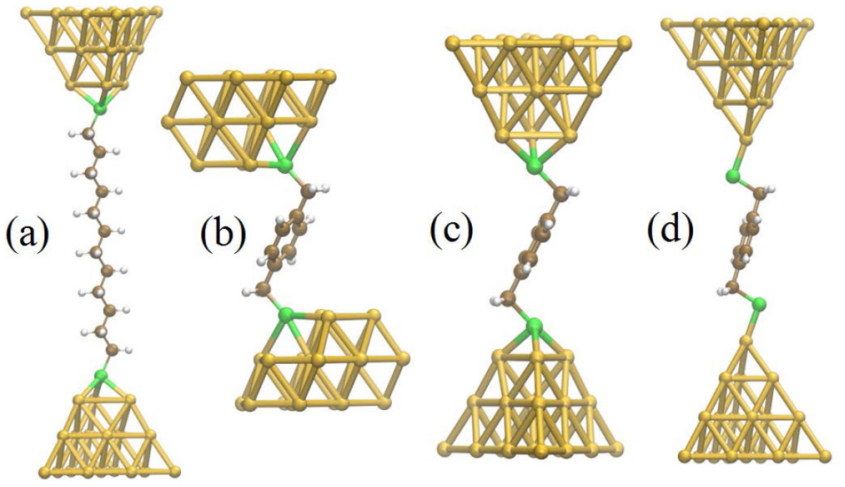

FIG. 6. (Color online) Representative dithiols studied theoretically, showing different models for adsorption sites on gold. (a) Dodecanedithiol bound at hollow sites, (b) $1 \mathrm{Ph} 1$ bound at step edge sites, (c) $1 \mathrm{Ph} 1$ bound at hollow sites, and (d) $1 \mathrm{Ph} 1$ bound at top sites.

understanding of the level alignment, which in turn impacts charge transport across the junction.

We constructed junctions in which each molecule is connected to two gold clusters, in the hollow, top, or step binding geometry (see Fig. 6), however, in what follows, we will restrict discussion to the hollow position. For comparison, we also studied a series of alkanedithiols of four different lengths ( $\mathrm{C} 2, \mathrm{C} 6, \mathrm{C} 8$, and $\mathrm{C} 12)$, also bound in the hollow position. Although their total lengths are different from the $x \operatorname{Ph} x$ molecules, this comparison was made to give insight into the presence of any additional state(s) and into differences in the length dependence. In Fig. 7, we show the low-bias transmission curves obtained for the $\mathrm{C} x$ and $x \mathrm{Ph} x$ series in the hollow geometry. For the $x \mathrm{Ph} x$ molecules, the presence of additional peaks is evident. These Breit-Wigner resonances approach the Fermi level as the molecular length increases (consistent with previous calculations $[55,56]$ ) and they do not appear in the case of the $\mathrm{C} x$ molecules. The presence of

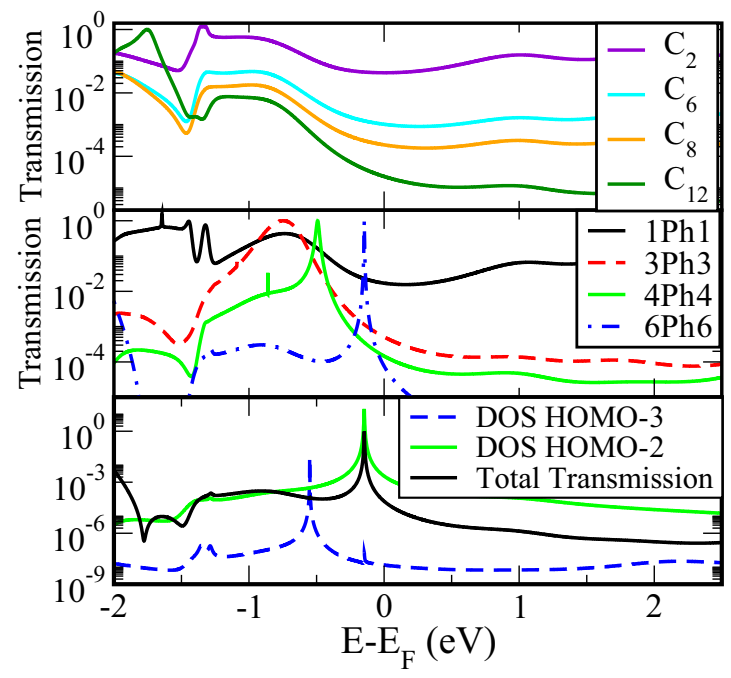

FIG. 7. (Color online) Transmission as a function of energy for $\mathrm{C} x$ and $x \mathrm{Ph} x$ in the hollow binding geometry and DOS projected onto HOMO-2 and HOMO-3 for 6Ph6 in the hollow geometry. 
these states creates an indentation in the barrier, confirming our earlier suggestion [18]. A similar effect has previously been shown in another system [57]. These resonances stem from the HOMO-2, which is localized on the benzene ring, thus they become narrower as the increasing benzene-gold distance decouples them from the gold contacts. To some extent, the benzene ring behaves as a quantum dot, weakly coupled to the electrodes by the alkyl chains. The trend in energy of the peaks approximately follows that in the gas phase for $3 \mathrm{Ph} 3,4 \mathrm{Ph} 4$, and $6 \mathrm{Ph} 6$, whereas the corresponding peak of $1 \mathrm{Ph} 1$ lines up further down in energy (at $-1.48 \mathrm{eV}$ ). Indeed, in the case of this shorter molecule, exceptional behavior is expected due to the strong hybridization of the orbitals. In the case of the $\mathrm{C} x$ molecules, the states closest to the Fermi level are localized mainly on the $\mathrm{S}$ atoms, while states localized on the whole chain appear only below $-2 \mathrm{eV}$.

To discern the different contributions in the transmission curves of the $x \mathrm{Ph} x$ molecules, we calculated the DOS projected onto the molecular orbitals. The results are illustrated for $6 \mathrm{Ph} 6$ in the lowest panel of Fig. 7. These projections clearly show that the sharp peak visible in the transmission curve close to the Fermi level for 6Ph6 is due to the HOMO-2, while the HOMO-3, also mainly localized on the benzene ring, does not yield any peak in the transmission curve apart from a very small Fano resonance (interference effects in transport through benzene rings have been widely studied [58]). The broad bumps at around $-1 \mathrm{eV}$ derive from the gateway states, i.e., from the hybridization of $\mathrm{S}-\mathrm{Au}$ states at the interface. Notice that using the same voltage $0.6 \mathrm{~V}$ as in the experiments is not expected to change the overall picture. To confirm this, we show, in the SM, a comparison between the transmission curves for $4 \mathrm{Ph} 4$ calculated at 0 and $0.6 \mathrm{~V}$ : the position of the HOMO-2 is not affected, while the bias voltage seems to rather have an influence on the interface states.

We now turn to analyze these systems quantitatively. In what follows, we will focus on the metal-molecule interface of our system in order to discern the various contributions that determine the level alignment in the studied junctions. We again focus on the hollow binding geometry. To begin with, we have first analyzed the Mulliken charges in order to discern whether any significant charge transfer takes place in this system (see Table I in the SM [29]). They show an increased electron charge (around 0.3) on each gold cluster and corresponding positive charge on the outermost carbon atoms, indicating charge transfer from the molecule onto the metal. Subsequently, we have analyzed a system consisting of each molecule connected to a single Au19 cluster in a hollow position, with the aim of understanding the level alignment at just one metal-molecule interface. In this case, as well as the standard DFT calculation, we have also performed constrained DFT (cDFT) calculations, as explained below. By connecting a Bethe lattice to the metal cluster, a new system is built, where the molecule is now connected to a semi-infinite electrode (see Ref. [42] for more details). The system can be hence divided into two parts, namely the lead and the main region that comprises the molecule and a certain number of metal layers. The density matrix of this region is calculated as

$$
P(\mu)=-\frac{2}{\pi} \operatorname{Im} \int_{-\infty}^{0} d E G^{+}(E ; \mu),
$$

where the retarded Green's function is given by

$$
G^{+}(E ; \mu)=[(E-\mu) S-H-\Sigma(E, \mu)]^{-1} .
$$

Here, $H$ is the Hamiltonian of the main region, $\Sigma$ is the lead self-energy, which describes the coupling to the semi-infinite lead, and $\mu$ is a quantity by which the on-site energies of the Hamiltonian must be shifted in order to ensure the total-charge neutrality ( $\mu$ is opposite in sign to the chemical potential). In our cDFT any charge transfer between the two parts is forbidden. Both surface and molecule were forced to keep a number of electrons as to maintain charge neutrality. To this aim, $\mu$ was computed in alternation so as to meet the imposed charge constraints on the metal and the molecule. If $N_{S}$ and $N_{\mathrm{mol}}$ are the desired number of electrons in the surface and the molecule, respectively, then two different chemical potentials $\mu_{S}$ and $\mu_{\mathrm{mol}}$ are calculated so that

$$
\begin{gathered}
\operatorname{Tr}\left[P_{S}\left(\mu_{S}\right) S_{S}\right]=\sum_{i, j=1}^{N A O_{S}} P(i, j) S(i, j)=N_{S}, \\
\operatorname{Tr}\left[P_{\mathrm{mol}}\left(\mu_{\mathrm{mol}}\right) S_{\mathrm{mol}}\right]=\sum_{i, j=N A O_{S}+1}^{N A O_{S}+N A O_{\mathrm{mol}}} P(i, j) S(i, j)=N_{\mathrm{mol}},
\end{gathered}
$$

where $N A O_{s}$ and $N A O_{\mathrm{mol}}$ are the number of atomic orbitals of the surface and the molecule, respectively. At each iteration step the density matrix $P^{o}$ is then built as a block matrix as follows:

$$
P^{o}=\left(\begin{array}{cc}
P_{S}\left(\mu_{S}\right) & P_{S-\mathrm{mol}} \\
P_{S-\mathrm{mol}} & P_{\mathrm{mol}}\left(\mu_{\mathrm{mol}}\right)
\end{array}\right) .
$$

The off-diagonal terms in the submatrices $P_{s \text {-mol }}$ were set to zero. The so-built density matrix is out of equilibrium, with two different chemical potentials in the metal and the molecule (at approximately $-5.96 \mathrm{eV}$ and $-4.2 \mathrm{eV}$ for metal and molecule, respectively), consequently giving rise to a step potential across the system. The Fermi level of the molecule calculated in this way can be interpreted as the charge neutrality level (CNL) discussed in the literature [46,59], but obtained in a more rigorous way. It defines the direction in which electronic charge is most likely to be transferred. In our case, the CNL is definitely higher than the Au Fermi level, so charge transfer from the molecule onto the metal is expected, confirming what was indicated by the Mulliken charges in the junction.

We now turn to analyze what happens once the electronic charge has been transferred. To this end, we have computed the on-site energies along the Au19-molecule junctions and compared them with those resulting from the constrained calculation (the results are shown in Fig. 8). They were evaluated as the average (over the atomic orbitals of each atom) of the diagonal terms in the Kohn-Sham Hamiltonian. Since the absolute values along the vertical axes are not physically relevant, as they are strongly related to the basis-set choice for each atom, they have been offset for clarity. Hence, we will only focus on the comparison between the values obtained in two kinds of calculations. At the interface, we see a clear sign of the expected dipole; in the unconstrained calculation the values are shifted upwards in the metal and downwards in the molecule. In the latter, the effect is stronger in the carbon atoms closer to the metal, fading away as the distance from the metal increases. In $1 \mathrm{Ph} 1$, the shortest molecule, the benzene 

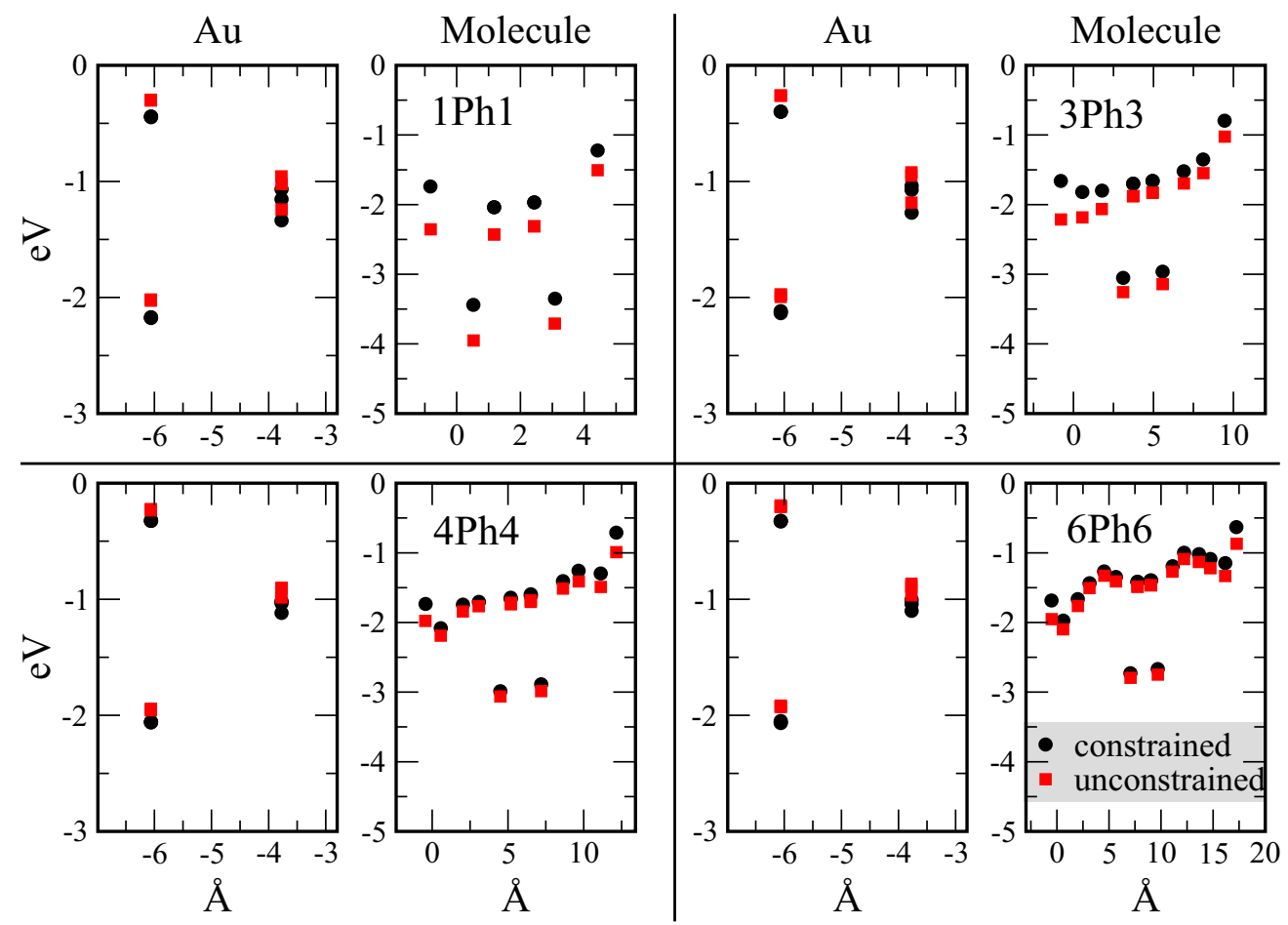

FIG. 8. (Color online) On-site energies (averaged over the atomic orbitals of each atom) in the three innermost Au atoms and the molecular $\mathrm{C}$ atoms in the $\mathrm{Au} 19-x \mathrm{Ph} x$ systems for all four molecules, in both constrained and unconstrained DFT.

ring is quite close to the gold and shows some positive charge (0.1) in the unconstrained calculation, as compared to that constrained. Indeed, for $1 \mathrm{Ph} 1$ the on-site energies are strongly affected also in the $\mathrm{C}$ atoms of the benzene ring. It is also worth adding that, when a second cluster is added at the other end of the molecules to complete the junctions, the effects caused by the dipole in the bulk region are expected to increase. The Mulliken charges along the Au19-molecule junctions in the constrained and unconstrained calculations are shown in the SM [29].

We summarize all these results as follows: when the $x \operatorname{Ph} x$ molecules approach the metal, Fermi-level alignment is achieved upon charge transfer from the sulfur, or the outermost carbon atoms, onto the metal. This generates a dipole potential mainly near the interface. The molecular bulk states (HOMO-2 levels) then behave like spectators, with their energetic positions being dragged down under the influence of the dipole potential according to the distance from the interface [Fig. 5(d)], and never crossing the Fermi level (see, for example, the case of the top binding geometry for the hypothetical $8 \mathrm{Ph} 8$ in the SM [29]). The level alignment of the bulk states is governed purely by electrostatics because of the clear separation from the interface (apart from 1Ph1) as already discussed. Note that this would not be possible in fully conjugated molecules, where other effects such as hybridization would also play a role. Notice that any electrostatic-induced shift is added to the one the HOMO-2 already presents in the gas phase for increasing chain length (see, in Fig. 9, a comparison between the energy shift experienced by this orbital in the gas phase and in the junction).

It should be recalled that there is some uncertainty in the position of the HOMO-2 peak in the transmission curves as it corresponds to a Kohn-Sham state, and it might be located at lower energies. Nevertheless, the computed transmission curves provide an insight into the alignment dynamics, i.e., they clearly show how the alignment of the bulk states is governed by the gas-phase behavior, modulated by what happens at the interface. To support our interpretation of the numerical results, it is important to verify that the benzene ring falls inside the space region in which the dipole-potential effect is confined. To this aim, we have calculated the potential generated in the middle of the benzene ring by the charges at the interface. The results are reported in Table II of the SM [29]

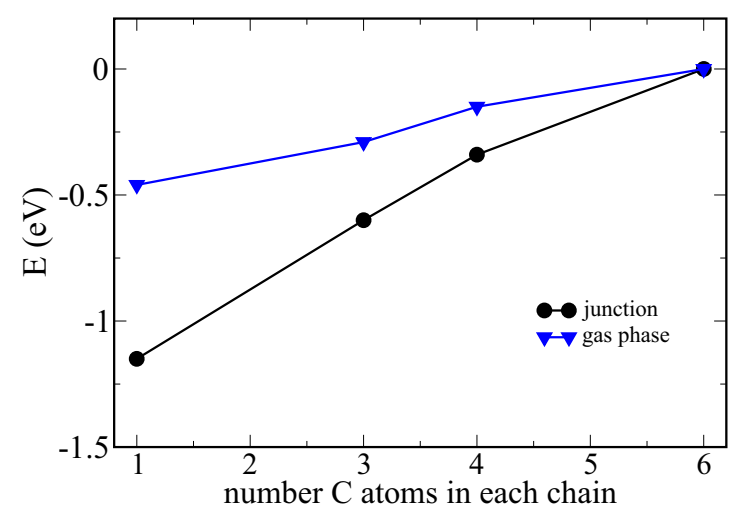

FIG. 9. (Color online) Energetic position of the HOMO-2 for each molecule in the junction and in the gas phase. All values have been shifted to set those for $6 \mathrm{Ph} 6$ to zero. It can be seen that the original displacement of the HOMO-2 in the gas phase increases in the junction. 


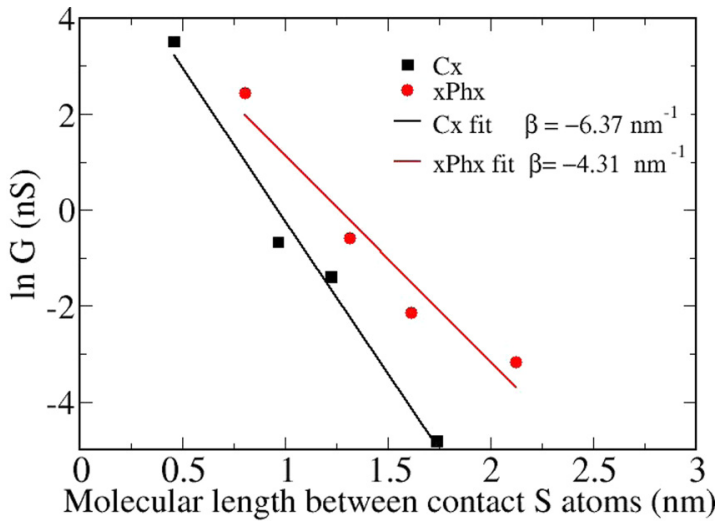

FIG. 10. (Color online) The calculated $\ln$ (conductance) as a function of the molecular length for $\mathrm{C} x$ and $x \mathrm{Ph} x$ in the hollow binding geometry

and show decreasing values for increasing chain length, with maximum values of around $1 \mathrm{eV}$.

Overall, the scenario described above provides a good rationalization for the experimentally observed low value of $\beta$. There are two counteracting effects, namely the expected decay due to increasing molecular length on the one hand, together with the increasing sharpness of the HOMO-2 resonance in the molecules with longer polymethylene bridges, and the approach to the Fermi energy of the resonance peak on the other hand. Figure 10 shows a plot of calculated zero bias conductance vs molecular length for the $x \mathrm{Ph} x$ and $\mathrm{C} x$ molecules. Although the absolute values of the conductances differ substantially from the experimentally observed values, it can be clearly seen that the calculated $\beta$ value is smaller for the $x \mathrm{Ph} x$ series than for the $\mathrm{C} x$ molecules, although the factor by which they differ (approximately 1.5) is substantially smaller than that seen experimentally (approximately 4.8). Neverthe- less, given the known problems with DFT, and the limitations inherent in the modeling of the contacts, we believe that this offers a good explanation for the experimental observations. Ultimately, possible asymmetries in the experimental junctions should be taken into account (not considered here) which lead to transmission resonances with a maximum height lower than 1. While this is not expected to change the conductance values for the $\mathrm{C} x$ molecules, for which the resonances show up further below the Fermi energy, it might change the conductance values for the $x \operatorname{Ph} x$ molecules and, consequently, the $\beta$ factor.

\section{CONCLUSIONS}

We studied the series of molecules $\mathrm{HS}\left(\mathrm{CH}_{2}\right)_{x}[1$, $\left.4 \mathrm{C}_{6} \mathrm{H}_{4}\right]\left(\mathrm{CH}_{2}\right)_{x} \mathrm{SH}(x=1,3,4,6)$ and found that the rate of conductance decay with molecular length is smaller than that measured previously for alkanedithiols. This was found to be due to the decreasing distance in energy of the Breit-Wigner resonance from the Fermi level for increasing molecular length. This trend originates from the orbital behavior in the gas phase but is modulated by the electrostatic balance established at the electrode contact region.

\section{ACKNOWLEDGMENTS}

This research was supported by the EPSRC (Grant No. EP/H035184/1), by MINECO under Grant No. FIS201347328, by the European Union structural funds and the Comunidad de Madrid MAD2D-CM Program under Grant. P2013/MIT-2850, and by Generalitat Valenciana under Grant PROMETEO/2012/011. We thank M. Soriano for help with the computational part and acknowledge the computer resources, technical expertise, and assistance provided by the Centro de Computación Científica of the Universidad Autónoma de Madrid.
[1] R. J. Nichols, W. Haiss, S. J. Higgins, E. Leary, S. Martin, and D. Bethell, Phys. Chem. Chem. Phys. 12, 2801 (2010).

[2] F. Chen, J. Hihath, Z. Huang, X. Li, and N. Tao, Annu. Rev. Phys. Chem. 58, 535 (2007).

[3] M. S. Hybertsen, L. Venkataraman, J. E. Klare, A. C. Whalley, M. L. Steigerwald, and C. Nuckolls, J. Phys.: Condens. Matter 20, 374115 (2008).

[4] F. Chen and N. Tao, Acc. Chem. Res. 42, 429 (2009).

[5] J. C. Cuevas and E. Scheer, Molecular Electronics: An Introduction to Theory and Experiment (World Scientific, Singapore, 2010).

[6] J. Park, A. N. Pasupathy, J. I. Goldsmith, C. Chang, Y. Yaish, J. R. Petta, M. Rinkoski, J. P. Sethna, H. D. Abruña, P. L. McEuen et al., Nature (London) 417, 722 (2002).

[7] J. Reichert, R. Ochs, D. Beckmann, H. Weber, M. Mayor, and H. von Löhneysen, Phys. Rev. Lett. 88, 176804 (2002).

[8] B. Xu and N. J. Tao, Science 301, 1221 (2003).

[9] S. Y. Quek, M. Kamenetska, M. L. Steigerwald, H. J. Choi, S. G. Louie, M. S. Hybertsen, J. Neaton, and L. Venkataraman, Nat. Nanotechnol. 4, 230 (2009).
[10] M. Kamenetska, S. Y. Quek, A. Whalley, M. Steigerwald, H. Choi, S. G. Louie, C. Nuckolls, M. Hybertsen, J. Neaton, and L. Venkataraman, J. Am. Chem. Soc. 132, 6817 (2010).

[11] F. Chen, X. Li, J. Hihath, Z. Huang, and N. Tao, J. Am. Chem. Soc. 128, 15874 (2006).

[12] L. Venkataraman, J. E. Klare, I. W. Tam, C. Nuckolls, M. S. Hybertsen, and M. L. Steigerwald, Nano Lett. 6, 458 (2006).

[13] S. Martín, W. Haiss, S. Higgins, P. Cea, M. C. Lopez, and R. J. Nichols, J. Phys. Chem. C 112, 3941 (2008).

[14] Y. S. Park, A. C. Whalley, M. Kamenetska, M. L. Steigerwald, M. S. Hybertsen, C. Nuckolls, and L. Venkataraman, J. Am. Chem. Soc. 129, 15768 (2007).

[15] R. Parameswaran, J. Widawsky, H. Vazquez, Y. Park, B. Boardman, C. Nuckolls, M. Steigerwald, M. Hybertsen, and L. Venkataraman, J. Phys. Chem. Lett. 1, 2114 (2010).

[16] W. Haiss, H. van Zalinge, S. J. Higgins, D. Bethell, H. Höbenreich, D. J. Schiffrin, and R. J. Nichols, J. Am. Chem. Soc. 125, 15294 (2003).

[17] W. Haiss, S. Martiín, E. Leary, H. v. Zalinge, S. J. Higgins, L. Bouffier, and R. J. Nichols, J. Phys. Chem. C 113, 5823 (2009). 
[18] E. Leary, S. J. Higgins, H. van Zalinge, W. Haiss, and R. J. Nichols, Chem. Commun. 43, 3939 (2007).

[19] G. K. Ramachandran, J. K. Tomfohr, J. Li, O. F. Sankey, X. Zarate, A. Primak, Y. Terazono, T. A. Moore, A. L. Moore, D. Gust et al., J. Phys. Chem. B 107, 6162 (2003).

[20] B. Q. Xu, X. L. Li, X. Y. Xiao, H. Sakaguchi, and N. J. Tao, Nano Lett. 5, 1491 (2005).

[21] R. Yamada, H. Kumazawa, T. Noutoshi, S. Tanaka, and H. Tada, Nano Lett. 8, 1237 (2008).

[22] S. K. Lee, R. Yamada, S. Tanaka, G. S. Chang, Y. Asai, and H. Tada, ACS Nano 6, 5078 (2012).

[23] E. J. Dell, B. Capozzi, K. H. DuBay, T. C. Berkelbach, J. R. Moreno, D. R. Reichman, L. Venkataraman, and L. M. Campos, J. Am. Chem. Soc. 135, 11724 (2013).

[24] G. Sedghi, L. J. Esdaile, H. L. Anderson, S. Martin, D. Bethell, S. J. Higgins, and R. J. Nichols, Adv. Mater. 24, 653 (2012).

[25] G. Sedghi, V. M. García-Suárez, L. J. Esdaile, H. L. Anderson, C. J. Lambert, S. Martín, D. Bethell, S. J. Higgins, M. Elliott, N. Bennett et al., Nat. Nanotechnol. 6, 517 (2011).

[26] G. Sedghi, K. Sawada, L. J. Esdaile, M. Hoffmann, H. L. Anderson, D. Bethell, W. Haiss, S. J. Higgins, and R. J. Nichols, J. Am. Chem. Soc. 130, 8582 (2008).

[27] Z. Li, T.-H. Park, J. Rawson, M. J. Therien, and E. Borguet, Nano Lett. 12, 2722 (2012).

[28] C. Wang, A. S. Batsanov, M. R. Bryce, S. Martín, R. J. Nichols, S. J. Higgins, V. M. García-Suárez, and C. J. Lambert, J. Am. Chem. Soc. 131, 15647 (2009).

[29] See Supplemental Material at http://link.aps.org/supplemental/ 10.1103/PhysRevB.91.195438 for details about synthetic procedures, single-molecule experiments, theoretical calculations, and spectra of compounds.

[30] W. Haiss, D. Lackey, J. K. Sass, and K. H. Besocke, J. Chem. Phys. 95, 2193 (1991).

[31] X. Xiao, B. Xu, and N. J. Tao, Nano Lett. 4, 267 (2004).

[32] E. Pires, J. E. Macdonald, and M. Elliott, Nanoscale 5, 9397 (2013).

[33] Z. Li, I. Pobelov, B. Han, T. Wandlowski, A. Błaszczyk, and M. Mayor, Nanotechnology 18, 044018 (2007).

[34] A. Busiakiewicz, S. Karthäuser, M. Homberger, P. Kowalzik, R. Waser, and U. Simon, Phys. Chem. Chem. Phys. 12, 10518 (2010).

[35] D. Fracasso, M. I. Muglali, M. Rohwerder, A. Terfort, and R. C. Chiechi, J. Phys. Chem. C 117, 11367 (2013).

[36] D. J. Wold, R. Haag, M. A. Rampi, and C. D. Frisbie, J. Phys. Chem. B 106, 2813 (2002).
[37] V. Kolivoska, M. Valasek, M. Gal, R. Sokolova, J. Bulickova, L. Pospisil, G. Meszaros, and M. Hromadova, J. Phys. Chem. Lett. 4, 589 (2013).

[38] M. J. Frish et al., Gaussian 09 Revision A.1, gaussian Inc. Wallingford CT, 2009, http://www.gaussian.com/ g_prod/g09.htm.

[39] J. P. Perdew, K. Burke, and M. Ernzerhof, Phys. Rev. Lett. 78, 1396 (1997).

[40] W. R. Wadt and P. J. Hay, J. Chem. Phys. 82, 284 (1985).

[41] J. J. Palacios, D. Jacob, A. J. Perez-Jimenez, E. S. Fabian, E. Louis, and J. A. Verges, ALACANT ab initio quantum transport package, see http://alacant.dfa.ua.es.

[42] D. Jacob and J. J. Palacios, J. Chem. Phys. 134, 044118 (2011).

[43] R. Ross, J. Powers, T. Atashroo, W. Ermler, L. LaJohn, and P. Christiansen, J. Chem. Phys. 93, 6654 (1990).

[44] J. C. Love, L. A. Estroff, J. K. Kriebel, R. G. Nuzzo, and G. M. Whitesides, Chem. Rev. 105, 1103 (2005).

[45] J. R. Widawsky, W. Chen, H. Vázquez, T. Kim, R. Breslow, M. S. Hybertsen, and L. Venkataraman, Nano Lett. 13, 2889 (2013).

[46] F. Flores, J. Ortega, and H. Vázquez, in Advances in Solid State Physics (Springer, New York, 2009), pp. 207-218.

[47] G. Heimel, L. Romaner, J.-L. Brédas, and E. Zojer, Surf. Sci. 600, 4548 (2006).

[48] M. Bokdam, D. Çakır, and G. Brocks, Appl. Phys. Lett. 98, 113303 (2011).

[49] O. T. Hofmann, D. A. Egger, and E. Zojer, Nano Lett. 10, 4369 (2010).

[50] S. Braun, W. R. Salaneck, and M. Fahlman, Adv. Mater. 21, 1450 (2009).

[51] R. Gutiérrez, G. Fagas, K. Richter, F. Grossmann, and R. Schmidt, Europhys. Lett. 62, 90 (2003).

[52] Y. Xue, S. Datta, and M. A. Ratner, J. Chem. Phys. 115, 4292 (2001).

[53] G. Peng, M. Strange, K. S. Thygesen, and M. Mavrikakis, J. Phys. Chem. C 113, 20967 (2009).

[54] F. Léonard and J. Tersoff, Phys. Rev. Lett. 84, 4693 (2000).

[55] F. Hüser, diploma thesis, Universität Karlsruhe, 2009.

[56] C. Benesch, M. Cizek, J. Klimeš, I. Kondov, M. Thoss, and W. Domcke, J. Phys. Chem. C 112, 9880 (2008).

[57] L. A. Zotti, E. Leary, M. Soriano, J. C. Cuevas, and J. J. Palacios, J. Am. Chem. Soc. 135, 2052 (2013).

[58] D. Darau, G. Begemann, A. Donarini, and M. Grifoni, Phys. Rev. B 79, 235404 (2009).

[59] H. Vázquez, F. Flores, and A. Kahn, IPAP Conf. Series 6, 1 (2005). 